\title{
Light-traffic analysis of queues with limited heterogenous retrials
}

\author{
Dieter Fiems \\ Ghent Univ., Dep. TELIN \\ St-Pietersnieuwstraat 41 \\ 9000 Gent, Belgium \\ Email: Dieter.Fiems@ugent.be
}

\author{
Tuan Phung-Duc \\ Faculty of Engineering, Information and Systems \\ University of Tsukuba \\ 1-1-1 Tennodai, Tsukuba, Ibaraki \\ 305-8573, Japan \\ Email: tuan@sk.tsukuba.ac.jp
}

\begin{abstract}
We consider a Markovian single-server retrial queueing system. Customers in orbit retry after an exponentially distributed amount of time, the retrial rate depending on the number of times the customer has retried before. Moreover, the number of retrials by a single customer is bounded by $K$, customers abandoning after the $k$ th retrial with probability $p_{k}$. As this queueing system is not amenable for exact analysis, we focus on its performance in light traffic. In particular, we describe a fast algorithm for calculating the terms in the Maclaurin series expansion of various performance measures, the arrival rate being the independent parameter of the expansion. We illustrate our approach by various numerical examples and verify the accuracy of the light traffic approximation by means of simulation.
\end{abstract}

\section{INTRODUCTION}

In a retrial queue, customers that cannot receive service immediately upon arrival join a virtual waiting room called orbit and retry getting service after some random time. As the total retrial rate depends on the number of customers in orbit, the underlying Markov chain of a retrial queue is not homogeneous. As a result, the analysis of retrial queues is often more difficult than similar models without retrials where customers wait in a queue at the server [1].

Retrial queueing models arise in various service systems such as call centers [8,9] and telecommunication systems such as cellular networks [7] and local area networks [3]. In conventional retrial queueing models, all retrial customers behave the same meaning that all blocked customers join the same orbit regardless of their number of retrials so far. In other words, the retrial intervals of customers are independent and identically distributed. This assumption significantly simplifies the problem as one only needs to track the number of retrying customers and the state of the servers.

In local area networks [3], a channel is shared by multiple terminals. If a terminal finds the channel busy, it

Permission to make digital or hard copies of all or part of this work for personal or classroom use is granted without fee provided that copies are not made or distributed for profit or commercial advantage and that copies bear this notice and the full citation on the first page. Copyrights for components of this work owned by others than the author(s) must be honored. Abstracting with credit is permitted. To copy otherwise, or republish, to post on servers or to redistribute to lists, requires prior specific permission and/or a fee. Request permissions from permissions@ acm.org.

QTNA '16, December 13 - 15, 2016, Wellington, New Zealand

(C) 2016 Copyright held by the owner/author(s). Publication rights licensed to ACM. ISBN 978-1-4503-4842-3/16/12 . .\$15.00

DOI: http://dx.doi.org/10.1145/3016032.3016056 will retransmit its packet at a later time. In particular, in CSMA/CD (carrier sense multiple access with collision detection) systems, after being blocked a first time, the node will retransmit its packet after a random time, uniformly distributed in $[0, a]$ where $a$ is some positive number. If upon retrying its packet is blocked again, the terminal node retransmits after a random time, again uniformly distributed, but in a wider range $[0,2 a]$. The retrial interval after being denied access twice will be uniformly distributed in $[0,4 a]$ and so on. Thus, the retrial behaviour of a packet depends on how many times it has been blocked. Motivated by this phenomenon, this paper investigates a retrial queueing model for CSMA/CD-like systems with multiple orbits, each orbit including customers with a specific number of retrials. Furthermore, if the number of retrials exceeds a threshold, we assume that the packet is lost.

Some contributions in literature relate to the model at hand. Shin and Moon [6, 5] consider a similar multiserver retrial queue with Poisson arrivals. Customers that are blocked for the first time either abandon the system or join the first orbit. If a retrial customer from the first orbit is blocked again it either abandons the system or joins the second orbit and so on. The number of retrials of a customer is also limited to $m$. The authors propose two iterative approximation methods for the model. Such iterative approximation methods are also applied to some specific retrial queueing networks [2]. The main idea is to decompose a complex model into several simpler sub-systems with unknown parameters and assume that these subsystems are independent. Furthermore, the inputs of one sub-system are computed from the outputs of other subsystems. Iteratively solving these subsystem one can get a convergence yielding the approximation to unknown parameters. One drawback of this method is that convergence is not rigorously proved.

Motivated by CSMA/CD-like systems, this paper focuses on a retrial queue with limited heterogeneous retrials like in $[6,5]$, but we assume arrivals come from a finite population. In contrast to previous work which investigates decomposition methods, we here consider the light-traffic approximation. Our method is a computational and allows for calculating the terms in the (light-traffic) series expansion efficiently. In light-traffic, the method can approximate the exact distribution with high accuracy at low computational complexity, the computational complexity of calculating the $T$ th term in the series expansion being $O\left(\min (T, N)^{K}\right)$ with $N$ the population size and $K$ the maximum number of retrials. 
The rest of our paper is organised as follows. Section 2 introduces the model and its notation in detail. Section 3 is then devoted to the light traffic analysis. Numerical results are presented in Section 4 while conclusions are drawn in Section 5 .

\section{MODEL}

We consider a single server retrial queue without buffer. There is a finite number $N$ of customers, each customer arriving at the system in accordance with a Poisson process with rate $\lambda$. Customers are served immediately if the server is available on arrival, the consecutive service times constituting a sequence of independent exponentially distributed random variables with common rate $\mu$.

If the server is busy upon arrival of a customer, the customer joins the orbit and retries after some time. If the customer finds the server free when he retries, he is served immediately. If not, the customer enters the orbit again with some probability or leaves the system. Customers retry at most $K$ times and leave the system for sure if they find the server occupied when they retry the $K$ th time. The time between retrials is exponentially distributed with rate $\gamma_{k}$ for a customer that already retried $k$ times, $k=0, \ldots, K-1$. In other words, $1 / \gamma_{k-1}$ is the mean time between the $k-1$ st and $k$ th retrial time (assuming that the customer retries $k$ times). Moreover, the probability that a customer leaves after unsuccessfully retrying also depends on the number of retrials so far. Let $h_{k}$ be the abandonment probability of a customer after $k+1$ retrials, $k=0, \ldots, K-1$, with $h_{K-1}=1$ as the customer abandons for sure after $K$ retrials.

Finally, we introduce some notation for further use. In the remainder, $\mathbf{e}$ is a vector of ones of size $K$, and $\mathbf{e}_{k}$ is a vector of zeroes of size $K$, apart from the $k$ th element which is 1 . For a vector, $\mathbf{x},|\cdot|$ denotes the $L^{1}$ norm, $|\mathbf{x}|=\sum_{k=0}^{K-1} x_{k}$.

\section{LIGHT TRAFFIC ANALYSIS}

As the customer retrial rate and the abandonment probabilities depend on the number of retrials so far, let $X_{k}(t)$ be the number of customers in orbit at time $t$ that have retried $k$ times and let $\mathbf{X}(t)$ be the vector with elements $X_{k}(t), k=0, \ldots, K-1$. Moreover, let $S(t) \in\{0,1\}$ be the number of customers in service at time $t$. In view of the modelling assumptions, it is easily verified that the process $\{(\mathbf{X}(t), S(t)), t \in \mathbb{R}\}$ constitutes a Markov process with state space

$$
\begin{aligned}
\mathcal{S} & =\left\{(\mathbf{x}, 0) \in \mathbb{N}^{K},|\mathbf{x}| \leq N\right\} \cup\left\{(\mathbf{x}, 1) \in \mathbb{N}^{K},|\mathbf{x}|<N\right\}, \\
& =\mathcal{S}_{i} \times\{0\} \cup \mathcal{S}_{a} \times\{1\},
\end{aligned}
$$

with $\mathcal{S}_{i}=\left\{\mathbf{x} \in \mathbb{N}^{K},|\mathbf{x}| \leq N\right\}$ and $\mathcal{S}_{a}=\left\{\mathbf{x} \in \mathbb{N}^{K},|\mathbf{x}|<\right.$ $N\}$. The definition of the state space above accounts for the observation that at most $N-1$ customers can be in orbit when a customer is being served.

For $\mathbf{X}(t)=\mathbf{x}=\left[x_{0}, x_{1}, \ldots, x_{K-1}\right]$ and $S(t)=1$, we have the following transition rates:

- The customer in service departs with rate $\mu$. The departure only changes the state of the server, the new system state being $(\mathbf{x}, 0)$.

- As each customer arrives in accordance with a Poisson process, a customer arrives with rate $\lambda(N-|\mathbf{x}|-1)$. As the server is busy, this customer joins the orbit, the new state being $\left(\mathbf{x}+\mathbf{e}_{0}, 1\right)$.
- Customers that have retried $k<K-1$ times, retry again without abandoning with rate $\gamma_{k} x_{k}\left(1-h_{k}\right)$. As the server is not available, the new state is $\left(\mathbf{x}-\mathbf{e}_{k}+\mathbf{e}_{k+1}, 1\right)$.

- Customers that have retried $k<K$ times, retry again with abandoning with rate $\gamma_{k} x_{k} h_{k}$. As the server is not available, the new state is $\left(\mathbf{x}-\mathbf{e}_{k}, 1\right)$.

For $\mathbf{X}(t)=\mathbf{x}=\left[x_{0}, x_{1}, \ldots, x_{K-1}\right]$ and $S(t)=0$, we have the following transition rates:

- As each customer arrives in accordance with a Poisson process, new customers arrive with rate $\lambda(N-|\mathbf{x}|)$. As the server is idle, the customer starts service, the new state being $(\mathbf{x}, 1)$.

- Customers that have retried $k<K$ times, retry again with rate $\gamma_{k} x_{k}$. As the server is available, the new state is $\left(\mathbf{x}-\mathbf{e}_{k}, 1\right)$.

With the summary of the state transitions above, we now introduce the balance equations. Let $\pi_{i}(\mathbf{x})$ and $\pi_{a}(\mathbf{x})$ denote the steady state probabilities to have $\mathbf{x}$ customers in orbit when the server is idle and active for $\mathbf{x} \in \mathcal{S}_{i}$ and $\mathbf{x} \in \mathcal{S}_{a}$. The balance equations then read,

$$
\pi_{i}(\mathbf{x})\left(\sum_{k=0}^{K-1} x_{k} \gamma_{k}+(N-|\mathbf{x}|) \lambda\right)=\pi_{a}(\mathbf{x}) \mu
$$

for $\mathbf{x} \in \mathcal{S}_{i}$ and,

$$
\begin{gathered}
\pi_{a}(\mathbf{x})\left(\sum_{k=0}^{K-1} x_{k} \gamma_{k}+(N-|\mathbf{x}|-1) \lambda+\mu\right)= \\
\quad \pi_{i}(\mathbf{x}) \lambda(N-|\mathbf{x}|)+\pi_{a}\left(\mathbf{x}-\mathbf{e}_{0}\right) \lambda(N-|\mathbf{x}|) \\
+\sum_{k=0}^{K-1} \pi_{i}\left(\mathbf{x}+\mathbf{e}_{k}\right) \gamma_{k}\left(x_{k}+1\right)+\sum_{k=0}^{K-2} \pi_{a}\left(\mathbf{x}+\mathbf{e}_{k}\right) \gamma_{k}\left(x_{k}+1\right) h_{k} \\
+\sum_{k=0}^{K-2} \pi_{a}\left(\mathbf{x}+\mathbf{e}_{k}-\mathbf{e}_{k+1}\right) \gamma_{k}\left(x_{k}+1\right)\left(1-h_{k}\right) \\
\quad+\pi_{a}\left(\mathbf{x}+\mathbf{e}_{K-1}\right) \gamma_{K-1}\left(x_{K-1}+1\right)
\end{gathered}
$$

for $\mathbf{x} \in \mathcal{S}_{a}$ with the normalisation condition,

$$
\sum_{\mathbf{x} \in \mathcal{S}_{a}} \pi_{a}(\mathbf{x})+\sum_{\mathbf{x} \in \mathcal{S}_{i}} \pi_{i}(\mathbf{x})=1
$$

For small $K$ and $N$, the balance equations can be easily solved. However, even for moderate $K$ and $N$, a direct computation of the stationary probabilities is computationally demanding, the size of the state space being approximately $2(N+K)^{K} / K$ !. For example for $N=100$ and $K=3$, there are nearly 350000 states.

Therefore, we study the system at hand in light-traffic. To this end, we introduce the Taylor series expansions of the stationary probabilities around $\lambda=0$,

$$
\pi_{a}(\mathbf{x})=\sum_{n=0}^{\infty} \pi_{a}^{(n)}(\mathbf{x}) \lambda^{n}, \quad \pi_{i}(\mathbf{x})=\sum_{n=0}^{\infty} \pi_{i}^{(n)}(\mathbf{x}) \lambda^{n}
$$

for $\mathbf{x} \in \mathcal{S}_{a}$ and $\mathbf{x} \in \mathcal{S}_{i}$ respectively. Before proceeding, we note that in the present setting it is easily verified (for example by applying Cramer's rule on the set of balance equations) that the stationary probabilities are rational functions of $\lambda$. As there is no singularity in $\lambda=0$, we conclude that 
the stationary probabilities are analytic functions in a region around $\lambda=0$ and the series expansion is justified.

Plugging the series expansions above in the balance equations and comparing terms in $\lambda^{n}$ yields,

$$
\begin{gathered}
\pi_{i}^{(n)}(\mathbf{x}) \sum_{k=0}^{K-1} x_{k} \gamma_{k}+\pi_{i}^{(n-1)}(\mathbf{x})(N-|\mathbf{x}|)=\pi_{a}^{(n)}(\mathbf{x}) \mu \\
\pi_{a}^{(n)}(\mathbf{x})\left(\sum_{k=0}^{K-1} x_{k} \gamma_{k}+\mu\right)=-\pi_{a}^{(n-1)}(\mathbf{x})(N-|\mathbf{x}|-1) \\
\quad+\pi_{i}^{(n-1)}(\mathbf{x})(N-|\mathbf{x}|)+\pi_{a}^{(n-1)}\left(\mathbf{x}-\mathbf{e}_{0}\right)(N-|\mathbf{x}|) \\
+\sum_{k=0}^{K-1} \pi_{i}^{(n)}\left(\mathbf{x}+\mathbf{e}_{k}\right) \gamma_{k}\left(x_{k}+1\right)+\sum_{k=0}^{K-2} \pi_{a}^{(n)}\left(\mathbf{x}+\mathbf{e}_{k}\right) \gamma_{k}\left(x_{k}+1\right) h_{k} \\
\quad+\sum_{k=0}^{K-2} \pi_{a}^{(n)}\left(\mathbf{x}+\mathbf{e}_{k}-\mathbf{e}_{k+1}\right) \gamma_{k}\left(x_{k}+1\right)\left(1-h_{k}\right) \\
\quad+\pi_{a}^{(n)}\left(\mathbf{x}+\mathbf{e}_{K-1}\right) \gamma_{K-1}\left(x_{K-1}+1\right)
\end{gathered}
$$

We can now recursively calculate all terms in the series expansion. For $n=0$ we have,

$$
\begin{gathered}
\pi_{i}^{(0)}(\mathbf{x}) \sum_{k=0}^{K-1} x_{k} \gamma_{k}=\pi_{a}^{(0)}(\mathbf{x}) \mu \\
\pi_{a}^{(0)}(\mathbf{x})\left(\sum_{k=0}^{K-1} x_{k} \gamma_{k}+\mu\right)=\sum_{k=0}^{K-1} \pi_{i}^{(0)}\left(\mathbf{x}+\mathbf{e}_{k}\right) \gamma_{k}\left(x_{k}+1\right) \\
+\sum_{k=0}^{K-2} \pi_{a}^{(0)}\left(\mathbf{x}+\mathbf{e}_{k}\right) \gamma_{k}\left(x_{k}+1\right) h_{k} \\
+\sum_{k=0}^{K-2} \pi_{a}^{(0)}\left(\mathbf{x}+\mathbf{e}_{k}-\mathbf{e}_{k+1}\right) \gamma_{k}\left(x_{k}+1\right)\left(1-h_{k}\right) \\
+\pi_{a}^{(0)}\left(\mathbf{x}+\mathbf{e}_{K-1}\right) \gamma_{K-1}\left(x_{K-1}+1\right)
\end{gathered}
$$

Plugging in any $\mathbf{x}$ with $|\mathbf{x}|=N$ in (3) shows $\pi_{i}^{(0)}(\mathbf{x})=0$. Iterating over $\mathcal{S}_{i} \backslash\{\mathbf{0}\}$ in reverse lexicographical order then further yields $\pi_{a}^{(0)}(\mathbf{x})=\pi_{i}^{(0)}(\mathbf{x})=0$ for $\mathbf{x} \in \mathcal{S}_{i} \backslash\{\mathbf{0}\}$. Moreover, evaluating (4) in $\mathbf{x}=\mathbf{0}$ shows $\pi_{a}^{(0)}(\mathbf{0})=0$. Finally, we invoke the normalisation condition,

$$
\sum_{\mathbf{x} \in \mathcal{S}} \pi_{i}^{(0)}(\mathbf{x})+\pi_{a}^{(0)}(\mathbf{x})=1
$$

to determine the unknown probability $\pi_{i}^{(0)}(\mathbf{0})=1$.

To determine the higher order terms, we solve (1) and (2) for $\pi_{a}^{(n)}(\mathbf{x})$ and $\pi_{i}^{(n)}(\mathbf{x})$, respectively. We have,

$$
\begin{gathered}
\pi_{a}^{(n)}(\mathbf{x})=\left(\sum_{k=0}^{K-1} x_{k} \gamma_{k}+\mu\right)^{-1}\left(-\pi_{a}^{(n-1)}(\mathbf{x})(N-|\mathbf{x}|-1)\right. \\
+\pi_{i}^{(n-1)}(\mathbf{x})(N-|\mathbf{x}|)+\pi_{a}^{(n-1)}\left(\mathbf{x}-\mathbf{e}_{0}\right)(N-|\mathbf{x}|) \\
+\sum_{k=0}^{K-1} \pi_{i}^{(n)}\left(\mathbf{x}+\mathbf{e}_{k}\right) \gamma_{k}\left(x_{k}+1\right)+\sum_{k=0}^{K-2} \pi_{a}^{(n)}\left(\mathbf{x}+\mathbf{e}_{k}\right) \gamma_{k}\left(x_{k}+1\right) h_{k} \\
+\sum_{k=0}^{K-2} \pi_{a}^{(n)}\left(\mathbf{x}+\mathbf{e}_{k}-\mathbf{e}_{k+1}\right) \gamma_{k}\left(x_{k}+1\right)\left(1-h_{k}\right) \\
\left.\quad+\pi_{a}^{(n)}\left(\mathbf{x}+\mathbf{e}_{K-1}\right) \gamma_{K-1}\left(x_{K-1}+1\right)\right)
\end{gathered}
$$

for $\mathbf{x} \in \mathcal{S}_{a}$ and,

$\pi_{i}^{(n)}(\mathbf{x})=\left(\sum_{k=0}^{K-1} x_{k} \gamma_{k}\right)^{-1}\left(\pi_{a}^{(n)}(\mathbf{x}) \mu-\pi_{i}^{(n-1)}(\mathbf{x})(N-|\mathbf{x}|)\right)$,

for $\mathbf{x} \in \mathcal{S}_{i}$. Close inspection of these expression reveals that the right-hand side contains lower order terms of $\pi_{a}$ and $\pi_{a}$ as well as terms of the same order, but for states which are lexicographically larger than $\mathbf{x}$. This implies that we can calculate the probabilities above in reverse lexicographical order for all $\mathbf{x} \in \mathcal{S}_{i} \backslash\{\mathbf{0}\}$. The expressions also allow to calculate $\pi_{a}^{(n)}(\mathbf{0})$. Finally, by the normalisation condition,

$$
\sum_{\mathbf{x} \in \mathcal{S}_{i}} \pi_{i}^{(n)}(\mathbf{x})+\sum_{\mathbf{x} \in \mathcal{S}_{a}} \pi_{a}^{(n)}(\mathbf{x})=0,
$$

one calculates $\pi_{i}^{(n)}(\mathbf{0})$ as follows for $n>0$,

$$
\pi_{a}^{(n)}(\mathbf{x})=-\sum_{\mathbf{x} \in \mathcal{S}_{i}} \pi_{i}^{(n)}(\mathbf{x})-\sum_{\mathbf{x} \in \mathcal{S}_{a} \backslash\{\mathbf{0}\}} \pi_{a}^{(n)}(\mathbf{x}) .
$$

The expressions above allow for determining the terms in the series expansions of $\pi_{i}(\mathbf{x})$ and $\pi_{a}(\mathbf{x})$. We can now calculate various performance measures of interest. For example, the $m$ th order expansion of the $k$ th order moment of the number of customers in orbit is,

$$
\begin{array}{r}
\mathrm{E}\left[|\mathbf{X}|^{k}\right]=\sum_{n=0}^{m}\left(\sum_{\mathbf{x} \in \mathcal{S}_{i}}|\mathbf{x}|^{k} \pi_{i}^{(n)}(\mathbf{x})+\sum_{\mathbf{x} \in \mathcal{S}_{a}}|\mathbf{x}|^{k} \pi_{a}^{(n)}(\mathbf{x})\right) \lambda^{n} \\
+O\left(\lambda^{m}\right) .
\end{array}
$$

REMARK 1. The numerical complexity of the former calculations is $O\left(N^{K} T\right)$ where $T$ is the number of terms. However, inspection of the recursion shows that the $T$ th terms in the series expansion of the stationary probabilities $\pi_{a}(\mathbf{x})$ and $\pi_{a}(\mathbf{x})$ are zero for $|\mathbf{x}|>T$. This observation is in line with the so-called $T$-events rule which says that in order to calculate the $T$ th term in the expansion, one only needs to consider sample paths with at most $T$ perturbations. By this observation, the computational complexity further reduces to $O\left(\min (T, N)^{K} T\right)$.

\section{NUMERICAL RESULTS}

To assess the accuracy of the light traffic approximations discussed above, we now consider some numerical examples. Figure 1 shows the mean content versus the product of the arrival rate and the number of customers for approximations of order $T=3, T=10$ and $T=30$ and for $N=10$, $N=100$ and $N=1000$ as indicated. All examples assume the following parameters: (i) Customers retry at most $K=3$ times; (ii) The retrial rates are $\gamma_{0}=0.5, \gamma_{1}=0.25, \gamma_{2}=$ 0.125 , which corresponds to mean retrial times of 2,4 and 8; and (iii) A customer leaves after retrying with probability $p_{0}=p_{1}=0.1$.

Each plot of figure 1 shows the $T$ th order approximation of the mean number of customers in orbit for $\mu=0.5$ (higher solid curve) and $\mu=1.0$ (lower solid curve). In addition, we have simulated the queueing system for assessing the accuracy, the dashed lines showing the simulation results.

The figure shows that our approximations are accurate for $\lambda N / \mu<0.8$, especially when we consider many terms in the series expansion. Moreover, for the 30th order approximation, we observe that our results are accurate up till 

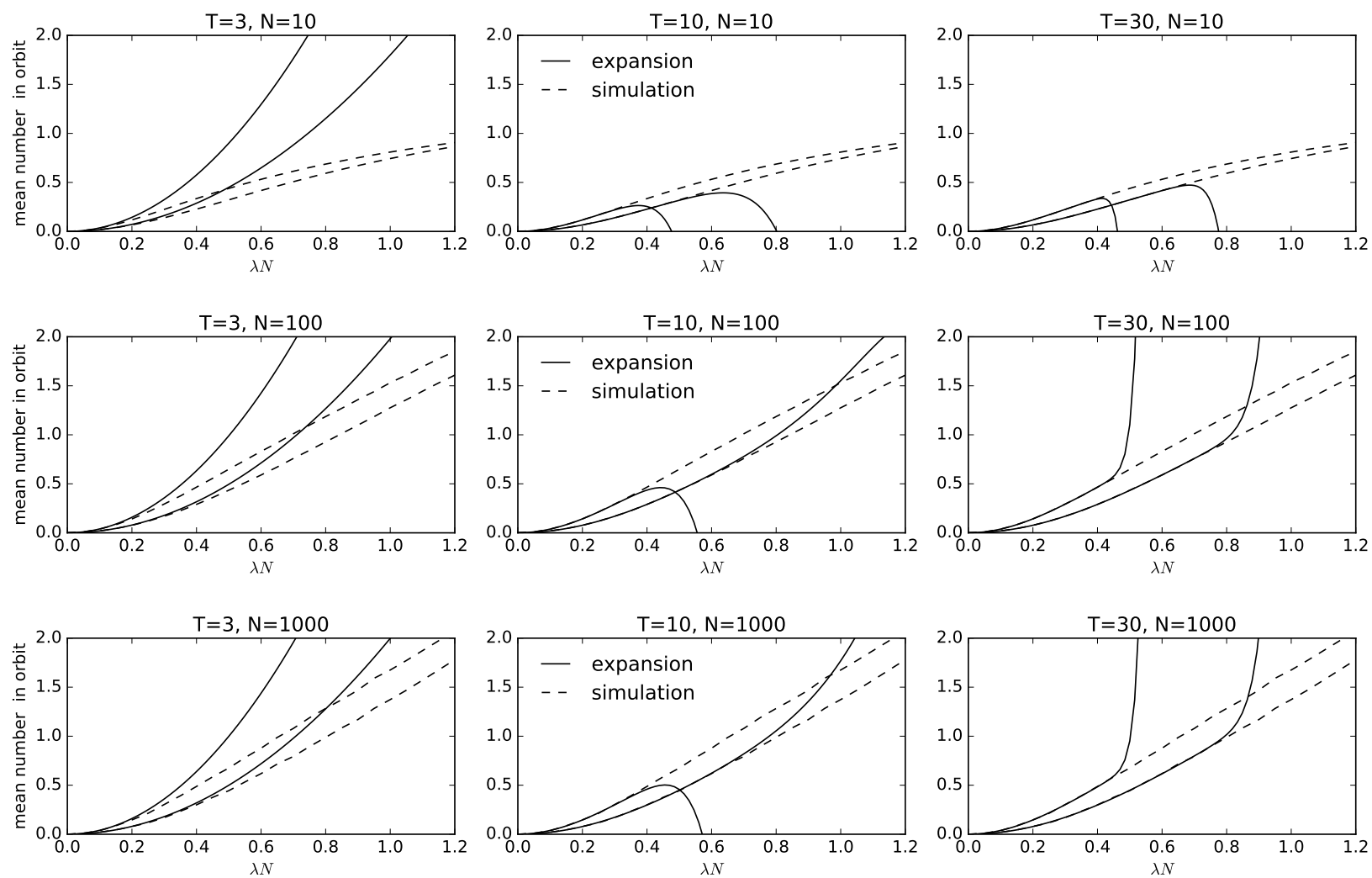

Figure 1: Tth order approximations of the mean number in orbit for various population sizes $N$.

a certain $\lambda_{\max }$ where the approximation quickly grows unbounded. This suggests that $\lambda_{\max }$ represents the radius of convergence of the series expansion. Higher order results not shown here, show that $\lambda_{\max }$ is approximately equal to $\lambda N / \mu$. This value is the maximum load the retrial queueing system would be able to serve, if the population was unbounded. Hence, it is not entirely unsurprising that the light-traffic approximation reaches its boundary: one cannot expect to assess an overloaded queue from its behaviour in light traffic. Finally, one observes that for $N$ sufficiently large, the population size hardly affects performance (assuming that the arrival rates are scaled with $N$ ). Indeed, it is not easy to see the difference between the plots for $N=100$ and $N=1000$.

\section{CONCLUDING REMARKS}

We presented the light traffic approximation for a retrial queue arising in the context of CSMA/CD. Our analysis yields accurate results at small computational complexity. In future work, we will adapt the decomposition method to the model under study and compare both approximations. In addition, we foresee that the light-traffic approximation can also be complemented with a fluid approximation to cover a wider area of the parameter space.

\section{REFERENCES}

[1] Falin, G. and Templeton, J. G. (1997). Retrial queues. CRC Press.

[2] Avrachenkov, K. and Yechiali, U. (2010). On tandem blocking queues with a common retrial queue.
Computers \& Operations Research, 37(7), 1174-1180.

[3] Choi, B. D., Shin, Y. W., and Ahn, W. C. (1992).

Retrial queues with collision arising from unslotted CSMA/CD protocol. Queueing systems, 11(4), 335-356.

[4] Artalejo, J. R. and Economou, A. (2005) On the non-existence of product-form solutions for queueing networks with retrials. Electronic Modeling 27, 13-19.

[5] Shin, Y. W. and Moon, D. H. (2010). Approximations of retrial queue with limited number of retrials. Computers \& Operations Research, 37(7), 1262-1270.

[6] Shin, Y. W. and Moon, D. H. (2008). Retrial queues with limited number of retrials: numerical investigations. In: Proc. of the 7th International Symposium on Operations Research and Its Applications (ISORA'08), 237-247.

[7] Tran-Gia, P. and Mandjes, M. (1997). Modeling of customer retrial phenomenon in cellular mobile networks. IEEE Journal on Selected Areas in Communications, 15(8), 1406-1414.

[8] Phung-Duc, T. and Kawanishi, K. (2011). Multiserver retrial queues with after-call work. Numerical Algebra, Control and Optimization, 1(4), 639-656.

[9] Phung-Duc, T. and Kawanishi, K. I. (2014). Performance analysis of call centers with abandonment, retrial and after-call work. Performance Evaluation, 80, 43-62. 